\title{
ORIGINAL
}

\section{ANÁLISIS COSTE-EFECTIVIDAD DEL PROGRAMA DE DETECCIÓN SISTEMÁTICA DEL CÁNCER CERVICAL EN LA REGIÓN DEL ALGARVE, PORTUGAL}

\section{Rosa María Novoa Vázquez}

Centro Regional de Saúde Pública. Administración Regional de Saúde del Algarve. Faro, Portugal.

\section{RESUMEN}

Fundamento: La evaluación económica de cuidados de salud es un instrumento de apoyo a la toma de decisión en la asignación de recursos entre diferentes alternativas. Ante la implementación de un programa organizado de detección sistemática masiva del cáncer cervical se realizó este estudio, cuyo objetivo fue evaluar el costoefectividad de tres alternativas: dos programas a implementar denominados «Detección con Pap» y «Detección con Thin-prep» y la estrategia actualmente en curso denominada «Detección oportunista».

Métodos: El análisis se realizó desde la perspectiva del Sistema Nacional de Salud. Se consideró un horizonte analítico de diez años. Se estimaron los costos directos médicos utilizando una tasa de actualización del $5 \%$. La efectividad fue estimada como el número de carcinomas detectados en fase preinvasora y el número de años de de carcinomas detectados en fase preinvasora y el número de años de
vida ganados. Se estimó la razón costo-efectividad para cada alternativa y el costo-efectividad incremental comparando cada alternativa a implementar con la alternativa actual. Se realizó un análisis de sensibilidad para las variables clave.

Resultados: El costo medio por carcinoma detectado fue de 1.199 euros para la «Detección con Pap», 3.148 euros para la «Detección oportunista» y 4.619 euros para la «Detección con Thin-prep». El costo medio por año de vida ganado fue 29 euros para la «Detección con Pap», 77 euros para la «Detección oportunista» y 114 euros para la «Detección con Thin-prep». La «Detección con Pap» tuvo un costo adicional de 623 euros por carcinoma adicional detectado y de 15 euros por año adicional de vida ganado. La «Detección con Thinprep» tuvo un costo adicional de 6.350 euros por carcinoma adicional detectado y de 156 euros por año adicional de vida ganado.

Conclusiones: La «Detección con Pap» tuvo la mejor relación costo-efectividad y el menor costo-efectividad incremental.

Palabras clave: Neoplasias de cuello uterino. Evaluación de programas. Análisis costo-beneficio. Asignación de recursos. Tamizaje masivo.

\section{Correspondencia:}

Rosa María Novoa Vázquez.

Rua poeta António Aleixo 32

Montenegro.

8000 Faro.

Portugal

Correo electrónico: rosamarianovoa@yahoo.es

\section{ABSTRACT}

\section{Cost-effectiveness of a cervical cancer screening programme in the Algarve region, Portugal}

Background: Economic evaluation of health care is an instrument of support to decision-making in the allocation of resources between different options. The current study was conducted with a view to implement an organised mass-screening programme. The objective was to evaluate the cost-effectiveness of three options: two programmes to be implemented that are called «Pap screening» and «Thin-prep screening», and the strategy currently in place called «Spontaneous screening».

Methods: The analysis was undertaken from the Health Care System perspective. The analytic horizon was 10 years. Direct medical costs were estimated and discounted at a rate of $5 \%$. Effectiveness was estimated as number of preinvasive carcinomas detected and life years gained. The cost-effectiveness ratio was estimated for the three options and incremental cost-effectiveness was estimated by comparison of the options to be implemented with the current strategy. A sensitivity analysis was conducted on the key variables.

Results: The average cost per carcinoma detected was 1,199 euros with «Pap screening», 3,148 euros with «spontaneous screening» and 4,619 euros with «Thin-prep screening». The average cost per life year gained was 29 euros with «Pap screening», 77 euros with «Spontaneous screening» and 114 euros with «Thin-prep screening». «Pap screening» had an additional cost of 623 euros per additional carcinoma detected and 15 euros per additional life year gained. «Thin-prep screening» had an additional cost of 6,350 euros per additional carcinoma detected and 156 euros per additional life year gained.

Conclusions: «Pap screening» had the best cost-effectiveness relation and the lowest additional cost-effectiveness.

Key words: Cervix neoplasms. Program evaluation. Cost-benefit analysis. Resource allocation. Mass screening. 


\section{INTRODUCCIÓN}

El cáncer cervical es una de las principales causas de muerte por cáncer en la mujer a nivel mundial. Es una causa de muerte evitable. El carcinoma de células escamosas, que representa entre el $85 \%$ y $90 \%$ de todos los carcinomas cervicales ${ }^{1}$, tiene un período de latencia prolongado. La mayoría de estos carcinomas puede ser prevenida mediante la detección precoz y el tratamiento de las lesiones escamosas intraepiteliales de alto grado.

Las tasas de supervivencia parecen estar asociadas al diagnóstico y tratamiento en estadios iniciales de la enfermedad. Las tasas esperadas a los cinco años son $100 \%$ para la neoplasia intraepitelial cervical preinvasora, $80 \%$ para el carcinoma invasor en estadio I y $10 \%$ para el carcinoma invasor en estadio IV ${ }^{2}$, lo que sugiere que cualquier medida que permita una detección precoz aumentará la supervivencia.

La experiencia de muchos países sugiere que son posibles reducciones importantes en la incidencia y mortalidad por cáncer cervical siguiendo programas organizados de detección sistemática ${ }^{3-7}$. La evidencia de la efectividad de estos programas se basa en estudios no experimentales ya que ningún ensayo aleatorizado controlado fue realizado hasta el momento. Los estudios revisados concluyen que son efectivos $\mathrm{y}$, aunque no aporten evidencia de clase I, constituyen un soporte para su adopción ${ }^{8-11}$.

La evaluación económica es una extensión necesaria a la evaluación de cuidados de salud y un instrumento de apoyo a la toma de decisión. Es una técnica complementaria recomendada cuando se decide la asignación de recursos entre diferentes alternativas. Es necesario identificar la más favorable y hasta que cantidad los beneficios adicionales justifican los costos adicionales.

En la región del Algarve (Portugal), contexto de este estudio, la estrategia de preven- ción del cáncer cervical consiste en la detección sistemática de oportunidad en los centros de salud, en el ámbito de las consultas de medicina familiar, sin que existan criterios explícitos. La Administración Regional de Salud consideró la posibilidad de implementar un programa organizado de detección sistemática masiva y por ello se realizó una evaluación económica.

El objetivo de este estudio es evaluar el costo-efectividad de tres alternativas de detección sistemática del cáncer cervical: dos programas de detección sistemática masiva aún no implementados y detección sistemática de oportunidad, alternativa actual.

\section{MATERIAL Y MÉTODOS}

\section{Descripción general del estudio}

Las cuestiones en estudio fueron:

- Identificar la alternativa de mejor relación costo-efectividad, como aquélla que tiene menor costo medio por unidad de resultado.

- Identificar la alternativa con menor costo-efectividad incremental, como aquélla que tiene menor costo adicional por unidad adicional de resultado.

Se estimaron los costos, los resultados y el costo-efectividad para cada alternativa, y el costo-efectividad incremental de cada alternativa a implementar en relación a la alternativa actual. El análisis se realizó desde la perspectiva del Servicio Nacional de Salud. Se consideró un horizonte analítico de diez años, periodo de 2003 a 2012.

Se modeló el resultado final de las tres alternativas, considerando como componentes del modelo la tasa de participación, la probabilidad de ocurrencia de cada procedimiento clínico y de cada tipo de lesión cito- 
lógica e histopatológica, y la sensibilidad del test de detección.

Se entendió como procedimiento clínico el test de citología, los tests de confirmación del diagnóstico, las diferentes técnicas de tratamiento, y las consultas de medicina familiar y ginecología.

Las alternativas consideradas fueron:

- Detección sistemática de oportunidad. Dirigida a las mujeres residentes en la región incluidas en el grupo de edad de 18-69 años. Intervalo de detección y seguimiento posterior sin criterios explícitos. El test es la citología con la técnica de Papanicolaou convencional. En este estudio denominada «Detección oportunista».

- Detección sistemática masiva con Papanicolaou. Dirigida a las mujeres residentes en la región incluidas en el grupo de edad de 30-64 años, con un intervalo de detección de tres años. El test es la citología con la técnica de Papanicolaou convencional. En este estudio denominada «Detección con Pap».

- Detección sistemática masiva con citología en capa fina (Thin-prep). Población objetivo e intervalo de detección igual a la anterior. El test es la citología en capa fina con la técnica de Papanicolaou en medio líquido. En este estudio denominada «Detección con Thin-prep».

Se consideró como intervalo de detección el periodo de tiempo que transcurre entre dos tests de detección consecutivos aplicados a cada mujer.

El seguimiento de los casos positivos a la citología en las alternativas de detección masiva puede verse en las figuras 1 y 2 , y el tratamiento en la tabla 1 . Se entendió como caso positivo todo resultado que no está dentro de los límites normales en los tests de citología y de confirmación del diagnóstico. Se utilizó la terminología del Sistema Bethesda para clasificar los resultados de la citología, la terminología de la Organización Mundial de la Salud para clasificar los resultados de la interpretación histopatológica y el estadío para el cáncer cervical de la Federación Internacional de Ginecología y Obstetricia.

Se entendió como tasa de participación la proporción de mujeres que realizarían el test cada año en relación al total de mujeres de la población objetivo anual. La población objetivo anual es constante y corresponde a la tercera parte del total de mujeres del grupo de edad definido para cada alternativa, según las estimaciones de la población femenina de la región para el año 2003. La población estimada para el grupo de 18-69 años es de 117.484 mujeres y para el grupo de 30-64 años es de 88.300 mujeres. En la alternativa de «Detección oportunista», la tasa de participación es del 25\% (el número esperado de mujeres que realizarán citología cada año es 9.678). En las dos alternativas de «Detección masiva» se utilizó una tasa de participación de $60 \%$ 12,13.

\section{Fuentes de información}

Los datos relativos a la población femenina por grupos de edad para el año 2003 para la región, la población femenina para el año 2000 para Portugal y la esperanza de vida por grupos de edad para el año 1999 para Portugal fueron obtenidos del Instituto Nacional de Estadística.

El número de casos de cáncer cervical estimado para Portugal para el año 2000 se obtuvo de la International Agency for Research on Cancer.

Los datos de la «Detección oportunista» del periodo de 1996 a 2001, relativos al número de mujeres que realizaron citología y 
edad de éstas fueron obtenidos del Servicio de Planificación de la Administración Regional de Salud del Algarve. La información relativa a los programas de detección sistemática a implementar y a los costos de divulgación, de formación de recursos humanos y del sistema de información que requeriría su implementación se obtuvo de la misma fuente.

Los datos de la «Detección oportunista» del periodo de 1996 a 2001, relativos al número de colposcopias, número de biopsias, diagnósticos histopatológicos, tratamientos realizados y edad de las mujeres con diagnóstico de neoplasia intraepitelial cervical proceden de los registros del Servicio de Ginecología de los hospitales de distrito de Faro y Portimão.

Los datos relativos a las probabilidades de ocurrencia de cada procedimiento clínico y de cada lesión citológica e histopatológica, utilizados para realizar los cálculos en las alternativas de detección masiva, provienen de la revisión bibliográfica sistemática de la literatura publicada ${ }^{2,14,21}$.

Los precios utilizados para valorar los costos unitarios de cada procedimiento clínico son los establecidos en la Tabla de precios para la facturación a los subsistemas de salud del Ministerio da Saúde.

\section{Procesamiento de la información}

Se utilizó el programa Statistical Package for the Social Sciences (SPSS) versión 10.0 para calcular el número de procedimientos esperado y la edad de detección de las neoplasias intraepiteliales cervicales en la alternativa de «Detección oportunista» y el programa Excel para calcular los costos.

\section{Cálculo del número anual de procedimientos clínicos en la alternativa de «Detección oportunista»}

Fue calculado a partir de los datos del periodo previo de 1996 a 2001. La tendencia de los valores observados fue determinada por regresión lineal simple (NC 95\%). Por ser uniforme para la colposcopia $(\mathrm{p}=0,675)$, biopsia $(\mathrm{p}=0,779)$, conización $(\mathrm{p}=0,880)$, curetaje endocervical ( $\mathrm{p}=0,221)$, histerectomía $(\mathrm{p}=0,270)$ y radioterapia $(\mathrm{p}=0,498)$, se utilizó la media aritmética de los valores observados. Estos valores son:

Tabla 1

Tratamiento según el tipo de lesión histopatológica

\begin{tabular}{|c|c|}
\hline Tipo de lesión & Tratamiento \\
\hline CIN I & Excisión electroquirúrgica \\
\hline CIN II/III & Conización + CEC \\
\hline Carcinoma microinvasor* & Histerectomía \\
\hline Carcinoma invasor* & Braquiterapia + Histerectomía \\
Estadio precoz & Radioterapia \\
Estadio avanzado & \\
\hline
\end{tabular}

Fuente: «Instituto Português de Oncologia» de Lisboa.CIN: Neoplasia Intraepitelial Cervical; CEC: Curetaje endocervical. *Carcinoma microinvasor: estadio 0; Estadio precoz: estadios I, II; Estadio avanzado: estadios III, IV. 
Figura 1

Fluxograma de confirmación diagnóstica de los casos positivos a la citología en la «Detección con Pap»

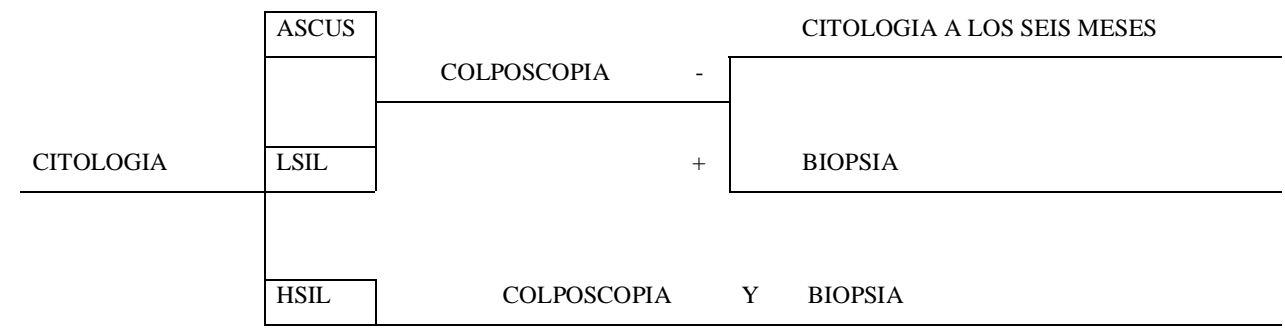

ASCUS: Células escamosas atípicas de significado indeterminado.

LSIL: Lesión intraepitelial escamosa de bajo grado.

HSIL: Lesión intraepitelial escamosa de alto grado.

Figura 2

Fluxograma de confirmación diagnóstica de los casos positivos a la citología en la «Detección con Thin-prep»

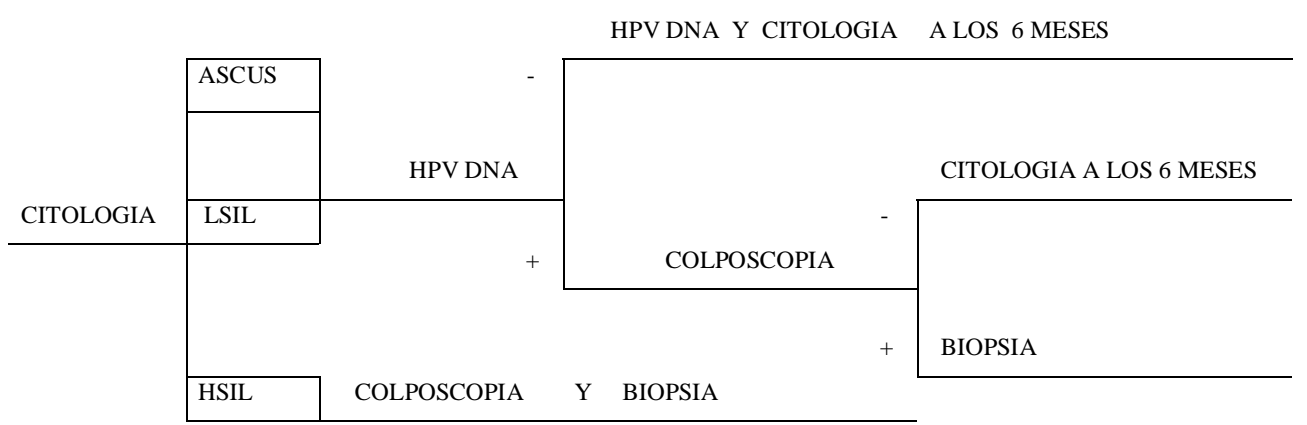

ASCUS: Células escamosas atípicas de significado indeterminado.

LSIL: Lesión intraepitelial escamosa de bajo grado.

HSIL: Lesión intraepitelial escamosa de alto grado.

HPV DNA: Test de tipificación del virus del papiloma humano

- colposcopia- 503,33 (DE 76,66)

- biopsia- 179,83 (DE 33,43)

- conización- 11,5 (DE 6,02)

- curetaje endocervical- 11 (DE 3,46)

- histerectomía- 6,67 (DE 4,37)

- radioterapia- $12,83(\mathrm{DE} 4,45)$

La tendencia no es uniforme para la citología $(\mathrm{p}=0,033)$ y la excisión electroquirúrgica $(\mathrm{p}=0,042)$. Para la citología se utilizó la media móvil ponderada de los valores observados, aplicando un modelo de serie temporal autorregresivo de orden 1-AR(1)- por ser el que mejor ajusta dados los valores observados en el periodo previo $(4.207,5.122$,

$5.841,6.022,6.852,6.010)$. El valor estimado es 9.678 (IC95\%: 6.038-13.323). Para la excisión electroquirúrgica, dados los valores observados en el periodo previo (2-2-0-0-00 ), se consideró el valor 0 .

\section{Cálculo del número anual de procedimientos clínicos en las alternativas de detección masiva}

Fue calculado a partir del fluxograma de decisión clínica (figuras 1 y 2) y de las probabilidades de ocurrencia extrapoladas de la literatura publicada (tabla 2), excepto el 
número de citologías que fue calculado a partir de la tasa de participación esperada y de la población objetivo anual.

\section{Cálculo de los costos}

Se consideraron los costos directos médicos. No se consideraron otros costos directos externos al servicio de salud, los costos indirectos, ni los costos intangibles. Se utilizó una tasa de actualización de $5 \%{ }^{22}$. Se calculó toda la secuencia de costos directos médicos que consumiría cada grupo de pacientes desde el momento de la detección para un horizonte temporal de diez años.

Se utilizaron los costos unitarios de cada procedimiento clínico y se valoraron de acuerdo con los precios publicados en la tabla para facturación a los subsistemas de Salud del Ministerio de Salud.

Corresponden a precios corrientes, en escudos del año 2001. El cambio a euros se hizo sobre la base de 1 euro igual a 200,482 escudos. Estos precios son:

- Examen citológico cervico-vaginal (Pap) - 3.330

- Examen citológico cervico-vaginal en medio líquido y con procesamiento automatizado en capa fina (Thin-prep) $-2.411$

- Test HPV DNA - 19.040

- Consulta de medicina familiar - 2.100

- Consulta de Ginecología - 3.100

- Biopsia - 4.830

- Excisión electroquirúrgica - 4.380

- Conización - 7.930

- Curetaje endocervical - 1.720

- Histerectomía - 1.079.766

- Braquiterapia - 452.111

- Radioterapia - 296.698

Para valorar el costo unitario del tratamiento con radioterapia se utilizó el precio del procedimiento codificado «intermedio» que incluye planeamiento intermedio, tomografía computarizada de planeamiento intermedio, simulación intermedia y tratamiento intermedio.

El costo total anual fue calculado como el sumatorio del costo unitario de cada procedimiento clínico multiplicado por el número anual de procedimientos. El costo total a los diez años fue calculado como el sumatorio del costo anual resultante de la aplicación de la tasa de actualización.

A cada citología con resultado positivo se le sumó el costo de una consulta de medicina familiar y a cada colposcopia el costo de una consulta de ginecología.

Se incluyeron también los costos de los tratamientos por carcinoma invasor que derivarían de las citologías con resultado falso negativo. Se asumió que todo resultado falso negativo progresaría a carcinoma y que estos carcinomas serían diagnosticados en un estadio avanzado y tratados con radioterapia. El número de resultados falsos negativos fue calculado a partir de la sensibilidad del test y de la tasa de prevalencia. Se utilizó una sensibilidad de $60 \%$ para el test Papanicolaou convencional $^{23,24}$ y de $90 \%$ para la citología en capa fina ${ }^{25}$. Se utilizó la tasa de prevalencia para Portugal para el año $2000(68,4$ por 100.000 mujeres), calculada a partir del número de casos estimado para Portugal para el año 2000 y de la población femenina estimada para Portugal para el mismo año.

En las dos alternativas de detección masiva se incluyeron los costos de divulgación por correo (carta de invitación para participar en el programa), divulgación (folletos informativos), formación profesional y sistema de información. Para los costos de correo se utilizó el costo unitario. Para la divulgación, formación y sistema de información se utilizó el costo total de operación en el primer año de implementación (250.000 euros). Se adicionaron 250.000 
euros al costo del primer año en las dos alternativas de detección masiva.

\section{Cálculo de los resultados}

La efectividad fue entendida como medida de resultado y calculada como el número de carcinomas detectados en fase preinvasora y de años de vida ganados.

Número de carcinomas detectados en fase preinvasora: se incluyeron en esta categoría las neoplasias intraepiteliales cervicales (CIN I, II, III). El número de CIN que serían detectadas cada año fue calculado utilizando la metodología descrita para el cálculo del número anual de procedimientos clínicos. El número de CIN que serían detectadas en el periodo de 2003 a 2012 fue calculado como el sumatorio de las detectadas cada año.
Para la alternativa de «Detección oportunista», el número anual de CIN fue calculado a partir del número de CIN detectadas en el periodo previo de 1996 a 2001. La tendencia de los valores observados, determinada por regresión lineal simple (NC 95\%), es uniforme $(\mathrm{p}=0,844)$. Se utilizó la media aritmética: 60,17 (DE 13,82).

Para las dos alternativas de detección masiva, el número anual de CIN fue calculado a partir del fluxograma de decisión clínica (figuras 1 y 2) y de las probabilidades de ocurrencia extrapoladas de la literatura (tabla 2).

Años de vida ganados: fue calculada la edad aproximada de muerte por carcinoma si no fuese implementado el programa, sumando el periodo de ventaja medio estimado para la detección sistemática del cáncer cer-

Tabla 2

Probabilidades de ocurrencia. Detección masiva

\begin{tabular}{|c|c|}
\hline Probabilidad & $\%$ \\
\hline $\begin{array}{c}\text { De lesiones en la citología }{ }^{14,15} \\
\text { HSIL } \\
\text { LSIL } \\
\text { ASCUS }\end{array}$ & $\begin{array}{c}0,4 \\
1,6 \\
4\end{array}$ \\
\hline $\begin{array}{c}\text { De CIN I en la biopsia }{ }^{16,18} \text { con diagnóstico histológico de: } \\
\text { HSIL } \\
\text { LSIL } \\
\text { ASCUS }\end{array}$ & $\begin{array}{c}4 \\
50 \\
30\end{array}$ \\
\hline $\begin{array}{c}\text { De CIN II/III en la biopsia }{ }^{16,18} \text { con diagnóstico citológico de: } \\
\text { HSIL } \\
\text { LSIL } \\
\text { ASCUS }\end{array}$ & $\begin{array}{c}95 \\
10 \\
8\end{array}$ \\
\hline $\begin{array}{c}\text { De la biopsia interpretar } \\
\text { Carcinoma microinvasor* } \\
\text { Carcinoma invasor }\end{array}$ & $\begin{array}{l}0,17 \\
0,23\end{array}$ \\
\hline $\begin{array}{c}\text { De carcinoma invasor por estadío } \\
\text { I } \\
\text { II } \\
\text { III } \\
\text { IV }\end{array}$ & $\begin{array}{c}38 \\
32 \\
26 \\
4\end{array}$ \\
\hline De colposcopia positiva $^{2}$ & 20 \\
\hline De HPV positivo $^{19,21}$ & 22 \\
\hline
\end{tabular}

HSIL: Lesión intraepitelial escamosa de alto grado. LSIL: Lesión intraepitelial escamosa de bajo grado. ASCUS: Células escamosas atípicas de significado indeterminado. CIN: Neoplasia intraepitelial cervical. HPV: Virus del papiloma humano. 
Tabla 3

Variables consideradas en el análisis de sensibilidad

\begin{tabular}{|c|c|cc|cc|}
\hline Variable & & \multicolumn{2}{|c|}{ Valores (\%) } & \\
\hline & Detección oportunista & \multicolumn{2}{|c|}{ Detección con Pap } & \multicolumn{2}{c|}{ Detección con Thin-prep } \\
\hline Tasa de participación & 50 & 70 & 50 & 70 & 50 \\
\hline Sensibilidad del test & 40 & 50 & 40 & 50 & 70 \\
\hline Tasa de actualización & 0 & 8 & 0 & 8 & 0 \\
\hline
\end{tabular}

vical a la edad en que éste sería identificado si fuese implementado el programa. Se asumió la esperanza de vida para el grupo de edad correspondiente como la estimación de los años de vida ganados por cada carcinoma detectado en fase preinvasora ${ }^{26}$. Multiplicando éstos por el número de carcinomas detectados en fase preinvasora en cada alternativa fueron obtenidos los años de vida ganados atribuidos a cada alternativa.

La edad en que sería identificado el carcinoma si fuese implementado el programa fue calculada como la mediana de las edades de las mujeres a quienes se detectó una neoplasia intraepitelial cervical con la «Detección oportunista» en el periodo previo de 1996 a 2001, ya que al aplicar el test Kolmogorv-Smirnov (NC 95\%) los valores observados no siguen una distribución normal $(\mathrm{p}=0,027)$. El valor estimado es 37 ( DQ 8).

Se utilizó el periodo de ventaja medio para la detección sistemática del cáncer cervical referido en la literatura de $5 \operatorname{años}^{27}$ y la esperanza de vida por grupos de edad para Portugal para el año 1999.

\section{Cálculo del costo-efectividad}

El costo-efectividad fue calculado como la razón entre los costos y los carcinomas detectados en fase preinvasora y como la razón entre los costos y los años de vida ganados. Fue expresado en términos de costo medio por carcinoma detectado en fase preinvasora y costo medio por año de vida ganado.
El costo-efectividad incremental fue calculado como la razón entre el costo adicional (diferencia de costos) y los carcinomas adicionales detectados en fase preinvasora (diferencia de carcinomas detectados), y como la razón entre el costo adicional y los años adicionales de vida ganados (diferencia de años de vida ganados), comparando cada alternativa de detección masiva con la alternativa de «Detección oportunista». Fue expresado en términos de costo adicional por carcinoma adicional detectado en fase preinvasora y de costo adicional por año adicional de vida ganado.

\section{Análisis de sensibilidad}

Se consideraron las variables tasa de participación, sensibilidad del test de detección y tasa de actualización. Se aplicó el análisis de «Situaciones extremas». Se utilizaron dos valores «extremos», en relación al valor considerado, excepto para la tasa de participación en la alternativa de «Detección oportunista», en la que se utilizaron valores superiores igualándolos a los de las alternativas de detección masiva y para la sensibilidad del test en la que se utilizaron valores inferiores (tabla 3). Se analizó el efecto de cada variable individualmente.

\section{RESULTADOS}

En la tabla 4 se presentan el costo, los carcinomas detectados en fase preinvasora, los años de vida ganados y el costo- efectividad 
Tabla 4

Costo, carcinomas detectados, años de vida ganados y costo-efectividad. Periodo 2003-2012

\begin{tabular}{|c|c|c|c|c|c|c|c|}
\hline Alternativa & $\begin{array}{c}\text { Costo } \\
\text { (euros) }\end{array}$ & $\begin{array}{c}\text { Ca } \\
\text { detectados }\end{array}$ & $\begin{array}{c}\text { Años de } \\
\text { vida ganados }\end{array}$ & $\begin{array}{c}\text { Costo- } \\
\text { efectividad* }\end{array}$ & $\begin{array}{c}\text { Costo- } \\
\text { efectividad** }\end{array}$ & $\begin{array}{c}\text { Costo- } \\
\text { efectividad } \\
\text { incremental* }\end{array}$ & $\begin{array}{c}\text { Costo- } \\
\text { efectividad } \\
\text { incremental** }\end{array}$ \\
\hline $\begin{array}{c}\text { Detección } \\
\text { oportunista }\end{array}$ & 1.888 .834 & 600 & 24.270 & 3.148 & 77 & & \\
\hline Detección con Pap & 3.154 .402 & 2.630 & $106.383,5$ & 1.199 & 29 & 623 & 15 \\
\hline $\begin{array}{c}\text { Detección con Thin- } \\
\text { prep }\end{array}$ & 5.127 .356 & 1.110 & $44.899,5$ & 4.619 & 114 & 6.350 & 156 \\
\hline
\end{tabular}

*por carcinoma detectado en fase preinvasora ** por año de vida ganado. Ca: Carcinomas

Figura 3

Años de vida ganados en cada alternativa. Periodo 2003 - 2012

DSP

106383,5

DST-P

44899,5

DO

DSP: Detección sistemática con Pap

DST-P: Detección sistemática con Thin-prep

DO: Detección oportunisata

para cada alternativa, para el periodo de 2003 a 2012. En la figura 3 se presentan los años de vida ganados en cada alternativa para el mismo periodo.

El costo medio por carcinoma detectado en fase preinvasora fue de 3.148 euros para la «Detección oportunista», 1.199 euros para la «Detección con Pap» y 4.619 euros para la «Detección con Thin-prep». El costo medio por año de vida ganado fue 77 euros para la «Detección oportunista», 29 euros para la «Detección con Pap» y 114 euros para la «Detección con Thin-prep».

El costo adicional de la «Detección con Pap», en relación a la «Detección oportunista», fue 623 euros por carcinoma adicional detectado en fase preinvasora y 15 euros por año adicional de vida ganado. El costo adicional de la «Detección con Thin-prep», en relación a la «Detección oportunista», fue 6.350 euros por carcinoma adicional detectado en fase preinvasora y 156 euros por año adicional de vida ganado.

\section{DISCUSIÓN}

La «Detección con Pap» es la alternativa de menor costo medio por carcinoma detectado en fase preinvasora y por año de vida ganado y la «Detección con Thin-prep» es la de mayor costo medio por carcinoma detectado en fase preinvasora y por año de vida ganado. En relación a la «Detección oportu- 
nista», la «Detección con Pap» tiene menor costo adicional por carcinoma adicional detectado en fase preinvasora y por año adicional de vida ganado que la «Detección con Thin-prep». La «Detección con Pap» es la alternativa de mejor relación costo-efectividad y con menor costo-efectividad incremental. Estas conclusiones no se modifican al variar la tasa de participación, la sensibilidad del test y la tasa de actualización.

Para una tasa de participación de $25 \%$ en la «Detección oportunista» y de $60 \%$ en las dos alternativas de detección masiva, la «Detección oportunista» es la que tiene menor costo y menos resultados. Cuesta 1.265.568 euros menos que la «Detección con Pap» y 3.238.521 euros menos que la «Detección con Thin-prep». Con la «Detección oportunista» se detectan 2.030 carcinomas menos y se ganan $82.113,5$ años de vida menos que con la «Detección con Pap», y se detectan 510 carcinomas menos y se ganan $20.629,5$ años de vida menos que con la «Detección con Thin-prep».

La «Detección con Pap» cuesta 1.972.953 euros menos que la «Detección con Thinprep», detecta 1.520 carcinomas más y gana 61.484 años de vida más que con la «Detección con Thin-prep».
En las dos alternativas de detección masiva, al disminuir la tasa de participación disminuyen el costo, el número de carcinomas detectados y de años de vida ganados, y aumenta el costo-efectividad (tabla 5). En las tres alternativas, al aumentar la tasa de participación aumentan el costo, el número de carcinomas detectados y de años de vida ganados, y disminuye el costo-efectividad (tabla 6).

Aumentar la tasa de participación mejora la relación costo-efectividad. Aumenta el número de carcinomas detectados en fase preinvasora y de años de vida ganados disminuyendo el costo medio por carcinoma detectado y por año de vida ganado.

En un análisis de costo-efectividad26, realizado en Dinamarca, que estima costos y efectos para una serie de programas existentes e hipotéticos, fue concluido que incrementar la tasa de participación gana años de vida adicionales sin incrementar el costo medio por año de vida ganado y consigue una mejor relación costo-efectividad que acortar el intervalo de detección o aumentar el segmento de edad de la población objetivo.

En la «Detección oportunista» pasar de una tasa de participación de $25 \%$ a $50 \%$ y de

Tabla 5

Costo, carcinomas detectados, años de vida ganados y costo-efectividad para una tasa de participación de $50 \%$. Periodo 2003-2012

\begin{tabular}{|c|c|c|c|c|c|c|c|}
\hline Alternativa & $\begin{array}{c}\text { Costo } \\
\text { (euros) }\end{array}$ & $\begin{array}{c}\text { Ca } \\
\text { detectados }\end{array}$ & $\begin{array}{c}\text { Años de } \\
\text { Vida ganados }\end{array}$ & $\begin{array}{c}\text { Costo- } \\
\text { efectividad* }\end{array}$ & $\begin{array}{c}\text { Costo- } \\
\text { efectividad* } \\
*\end{array}$ & $\begin{array}{c}\text { Costo- } \\
\text { efectividad } \\
\text { incremental } \\
*\end{array}$ & $\begin{array}{c}\text { Costo- } \\
\text { efectividad } \\
\text { incremental** }\end{array}$ \\
\hline $\begin{array}{c}\text { Detección } \\
\text { oportunista }\end{array}$ & 3.629 .338 & 1.174 & $47.488,3$ & 3.091 & 76 & -2.854 & -70 \\
\hline Detección con Pap & 2.677 .930 & 2.190 & $88.585,5$ & 1.222 & 30 & -936 & -23 \\
\hline $\begin{array}{c}\text { Detección con Thin- } \\
\text { prep }\end{array}$ & 4.325 .900 & 930 & $37.618,5$ & 4.651 & 114 & & \\
\hline
\end{tabular}

*por carcinoma detectado en fase preinvasora **por año de vida ganado. Ca: Carcinomas 
Tabla 6

Costo, carcinomas detectados, años de vida ganados y costo-efectividad para una tasa de participación de $70 \%$. Periodo 2003-2012

\begin{tabular}{|c|c|c|c|c|c|c|c|}
\hline Alternativa & $\begin{array}{c}\text { Costo } \\
\text { (euros) }\end{array}$ & $\begin{array}{c}\mathrm{Ca} \\
\text { detectados }\end{array}$ & $\begin{array}{c}\text { Años de } \\
\text { vida ganados }\end{array}$ & $\begin{array}{c}\text { Costo- } \\
\text { efectividad* }\end{array}$ & $\begin{array}{c}\text { Costo- } \\
\text { efectividad* } \\
*\end{array}$ & $\begin{array}{c}\text { Costo- } \\
\text { efectividad } \\
\text { incremental } \\
*\end{array}$ & $\begin{array}{c}\text { Costo- } \\
\text { efectividad } \\
\text { incremental** }\end{array}$ \\
\hline $\begin{array}{c}\text { Detección } \\
\text { oportunista }\end{array}$ & 5.055 .122 & 1.644 & $66.499,8$ & 3.074 & 76 & -2.618 & -64 \\
\hline $\begin{array}{c}\text { Detección con Pap } \\
\text { Detección con Thin- } \\
\text { prep }\end{array}$ & 5.585 .425 & 3.070 & $124.181,5$ & 1.167 & 28 & -1.030 & -25 \\
\hline
\end{tabular}

*por carcinoma detectado en fase preinvasora **por año de vida ganado. Ca: Carcinomas

$50 \%$ a $70 \%$ tiene un costo adicional de 3.000 euros por carcinoma adicional detectado $\mathrm{y}$ de 75 euros por año adicional de vida ganado. En la «Detección con Pap» pasar de una tasa de participación de $50 \%$ a $70 \%$ tiene un costo adicional de 1.000 euros por carcinoma adicional detectado y de 25 euros por año adicional de vida ganado. En la «Detección con Thin-prep» pasar de una tasa de participación de $50 \%$ a $70 \%$ tiene un costo adicional de 4.000 euros por carcinoma adicional detectado y de 100 euros por año adicional de vida ganado.

Al igualar la tasa de participación en las tres alternativas (tablas 5 y 6), el costo de la «Detección oportunista» es superior al de la «Detección con Pap» y tiene menos resultados que ésta. El costo-efectividad incremental de la «Detección con Pap» es «negativo», lo que implica que ahorra euros por cada carcinoma adicional detectado y por cada año adicional de vida ganado en relación a la «Detección oportunista».

La «Detección oportunista» se dirige a mujeres incluidas en un segmento de edad más amplio, con un intervalo de detección no explícito. Es posible que haya mujeres que realicen citología anualmente y que una proporción de éstas se incluyan en el grupo de 18-30 años, dado el contexto en que se aplica. Esto tendría un efecto importante en el aumento de costos y pequeño en el número de años de vida ganados y podría explicar que, al igualar la tasa de participación, detecte menos neoplasias intraepiteliales cervicales y gane menos años vida con un costo superior que la «Detección con Pap».

Comparando la «Detección oportunista» y la «Detección con Thin-prep», la «Detección oportunista» detecta más carcinomas y gana más años de vida con un costo inferior, por lo que ahorra euros por cada carcinoma adicional detectado y por cada año adicional de vida ganado. En la «Detección con Thinprep», el seguimiento planteado tiene un costo alto y podría influir en el menor número de neoplasias intraepiteliales cervicales detectadas y de años de vida ganados, en relación a las otras alternativas.

Otros estudios revisados ${ }^{7,28,29}$ concluyen que es más eficiente aumentar el número de años de vida ganados consiguiendo una alta cobertura de las mujeres incluidas en grupos de edad de alto riesgo que aumentar el segmento de edad de la población objetivo o acortar el intervalo de detección.

En las tres alternativas al disminuir la sensibilidad del test aumentan el costo y el costoefectividad. El número de carcinomas detec- 
tados y de años de vida ganados no varía. La sensibilidad del test tiene influencia en los costos derivados de los resultados falsos negativos $\mathrm{y}$, por tanto, del tratamiento con radioterapia. El aumento en el costo y, en consecuencia, en el costo-efectividad es proporcional a la disminución de la sensibilidad.

En las tres alternativas aumentan el costo y el costo-efectividad si no se aplica tasa de actualización. Si se aumenta esta tasa disminuyen el costo y el costo-efectividad. En ambas situaciones el número de carcinomas detectados y de años de vida ganados no varía.

Limitaciones a los resultados del estudio:

En la alternativa de «Detección oportunista», hacer predicciones para un futuro de diez años a partir de datos de un periodo previo de seis, introduce un sesgo en los cálculos del número de procedimientos clínicos y del número de neoplasias intraepiteliales cervicales detectadas. En las dos alternativas de detección masiva, considerar probabilidades de ocurrencia extrapoladas de la literatura para hacer las predicciones para el futuro, introduce un sesgo en los resultados de los cálculos del número de procedimientos clínicos y de neoplasias intraepiteliales cervicales detectadas. Considerar que todas las neoplasias intraepiteliales cervicales progresan a carcinoma, contabilizando las detectadas en el resultado final, introduce un sesgo que sobrestima la medida de efectividad de las tres alternativas. Considerar valores de referencia como costos unitarios de cada procedimiento repercute en el cálculo de los costos. Los valores corresponden a precios y no propiamente a costos, ya que integran los costos de los componentes, el valor de los recursos humanos y materiales requeridos en cada actividad. Considerar que todo resultado falso negativo progresa a carcinoma invasor en estadio avanzado y contabilizar desde el primer año de implementación los costos de estos carcinomas introduce un sesgo que sobrestima los costos del tratamiento con radioterapia. Esto reper- cute más en las alternativas que utilizan el test Papanicolaou, por tener menor sensibilidad y mayor tasa de falsos negativos. No incluir los costos directos no médicos ni los costos indirectos subestima los costos totales. Teniendo en cuenta que la evaluación se hace desde la perspectiva de los servicios de salud y no desde una perspectiva social, esta limitación puede ser minimizada.

Sería importante considerar la proporción de lesiones preinvasoras que evolucionan a carcinoma invasor, la cuantificación temporal de la progresión y las variables que la condicionan, así como el impacto en la calidad de vida del programa de detección. La medida de los beneficios y posibles efectos desfavorables de la detección no es completa sin tener en cuenta su efecto en la calidad de vida.

\section{AGRADECIMIENTOS}

A Ana María Gálvez, Departamento de Economía de la Salud de la Escuela Nacional de Salud Pública de La Habana, Cuba y a José Luis Otero, Unidad de Bioestadística de la Facultad de Medicina de Santiago de Compostela, España.

\section{BIBLIOGRAFÍA}

1. Brinton LA, Fraumeni JF. Epidemiology of Uterine Cervical Cancer. J Chronic Dis.1986; 39: 1051-65.

2. Austoker J, McPherson A. Cervical Screening. $2^{\mathrm{a}}$. ed. New York: Oxford University Press; 1992.

3. Knox EG. Cancer of the uterine cervix. En: Magnus $\mathrm{K}$, editor. Trends in cancer incidence. New York: Hemisphere Publishing corporation; 1982. p. 271-7.

4. Hakama M. Trends in the incidence of cervical cancer in the nordic countries. En: Magnus K, editor. Trends in cancer incidence. New York: Hemisphere Publishing corporation; 1982. p. 279-92.

5. Miller AB. The canadian experience of cervical cancer: Incidence trends and planned natural history investigation. En: Magnus K, editor. Trends in

Rev Esp Salud Pública 2004, Vol. 78, N. ${ }^{\circ} 3$ 
cancer incidence. New York: Hemisphere Publishing corporation; 1982. p. 311-20.

6. Hristova L, Hakama M. Effect of screening in the Nordic countries on deaths, costs and quality of life up to the year 2017. Acta Oncol 1997; 36:1-60.

7. Parkin DM, Moss SM. An evaluation of screening policies for cervical cancer in England and Wales using a computer simulation model. J Epidemiol Community Health 1986; 40:143-53.

8. Sasiensi P, Adams J. Effect of screening on cervical cancer mortality in England and Wales: analysis of trends with an age period cohort model. BMJ 1999; 318:1244-5.

9. Herrero R, Brinton LA, Reeves WC, Brenes MM, De Britton RC, Gaitan E et al. Screening for Cervical Cancer in Latin America: a case-control study. Int J Epidemiol 1992; 21:1050-6.

10. Van der Graaf Y, Zielhuis GA, Peer PG, Vooijs PG. The effectiveness of cervical screening: a population-based case-control study. J Clin Epidemiol 1988; 41:21-6.

11. Boyes DA, Morrison B, Knox EG, Draper GJ, Miller AB. A cohort study of cervical cancer screening in British Columbia. Clin Invest Med 1982; 5:1-29.

12. Hogod C, Fog J. Screening- Why, when and how? Denmark: National Board of Health; 1991.

13. De Novo Establishment and Cost-effectiveness of Papanicolaou Cytology Screening Service in the Socialist Republic of Vietnam. Cancer 2001; 91:928-39.

14. Davey DD, Naryshkin S, Nielsen ML, Kline TS. Atypical squamoux cells of undetermined significance: interlaboratory comparison and quality assurance monitors. Diagn cytopathol 1994; 11:390-6.

15. Davey DD, Woodhause S, Styer P, Stastny J, Mody D. Atypical epithelial cells and specimen adequacy. Arch Pathol Lab Med 2000; 124:203-11.

16. Chesebro MJ, Everet WD. A Cost-benefit Analysis of Colposcopy for Cervical Squamous Intraepitelial Lesions Found on Papanicolaou Smear. Arch Fam Med. 1996; 5:576-81.

17. Ostor AG. Natural history of cervical intraepitelial neoplasia: a critical review. Int J Gynecol Pathol 1993; 12:186-92.
18. Rabb SS, Steiner AL, Hornberger J. The costeffectiveness of treating women with a cervical vaginal smear diagnosis of atypical squamous cells of undetermined significance. Am J Obstet Gynecol 1998; 179:411-20.

19. Denny L, Kuhn L, Pollack A, Wainwright H, Wright TC. Evaluation of alternative methods of cervical cancer screening for resource-poor settings. Cancer. 2000; 89:826-33.

20. Hatch KD, Fu YS. Cáncer cervical y vaginal. En: Berek JS, Hillard PA, Adashi EY, editores. Ginecología de Novak. 12a . ed. Mexico: McGraw-Hill Interamericana; 1997.p. 1111-53.

21. Cuzick J, Sasiensi P, Davies P, Adams J, Normand $\mathrm{C}$, Frater A et al. A systematic review of the role of human papillomavirus testing within a cervical screening programme. Health Technol Assess 1999; 3:1-196.

22. Haddix AC, Shaffer PA. Cost-effectiveness Analysis. En: Haddix AC, Teutsch S, Shaffer PA, Duñiet DO, editores. Prevention effectiveness: A guide to decision analysis and economic evaluation. $1^{a}$. ed. New York: Oxford University Press; 1996. p. 103-27.

23. Nanda K, McCrory DC, Myers ER, Bastian LA, Hasselblad V, Hickey JD et al. Accuracy of the Papanicolaou test in screening for and follow-up of cervical cytologic abnormalities: a sistematic review. Ann Intern Med. 2000; 132:810-9

24. Fahey MT, Irving L, Macaskill P. Meta-analysis of Pap test accuracy. Am J Epidemiol 1995; 141:680-9.

25. Myers ER, McCrory DC, Subramanian S. Setting the target for a better cervical screening test: characteristics of a cost-effective test for cervical neoplasia screening. Obstet Gynecol 2000; 96:645-52.

26. Gyrd- Hansen D, Hdund B, Anderson P. A costeffectiveness analysis of cervical cancer screening: health policy implications. Health Policy 1995; $34: 35-51$.

27. Jenniceck M. Epidemiología. La lógica de la medicina moderna. $1^{\mathrm{a}}$. ed. Barcelona: Masson; 1996.

28. Koopmanschap MA, Van Oortmarssen GJ, Van Agt HM, Van Ballegooijen M, Habbema JD, Lubbe KT. Cervical-cancer screening: attendance and costeffectiveness. Int J Cancer 1990; 45:410-5.

29. Bos AB, Van Ballegooijen M, Van Gessel-Dabekaussen AA, Habbema JD. Organised cervical cancer screening still leads to higher coverage than spontaneous screening in the Netherlands. Eur J Cancer 1998; 34:1598-601. 